\title{
Manifestations of left ventricular dysfunction and arrhythmia in patients with chronic hypoparathyroidism and pseudohypoparathyroidism: a preliminary study
}

Yabing Wang ${ }^{1+}{ }^{\mathbb{D}}$, Kun $\mathrm{He}^{2+}$, Ou Wang ${ }^{1}$, Xue Lin ${ }^{3 *+}$, Sixing Chen ${ }^{1}$, Yan Jiang ${ }^{1}$, Mei $\mathrm{Li}^{1}$, Weibo Xia ${ }^{1}$ and Xiaoping Xing ${ }^{1 *+}$

\begin{abstract}
Background: Cardiac damage triggered by severe hypocalcemia is well known. However, the role of chronic hypoparathyroidism (HP) and pseudohypoparathyroidism (PHP) in cardiac health is still unclear. We investigated the effect of chronic HP and PHP on cardiac structure and conductive function in patients compiling with treatment.

Methods: The study included 18 patients with HP and eight with PHP aged $45.4 \pm 15.4$ and $22.1 \pm 6.4$ years, respectively with a previously regular follow-up. In addition, 26 age- and sex-matched healthy controls were included. General characteristics and biochemical indices were recorded. Cardiac function and structure were assessed by estimation of myocardial enzymes, B-type natriuretic peptide (BNP), and echocardiography. The 12-lead electrocardiogram and 24-h Holter electrocardiography were performed to evaluate the conductive function.

\footnotetext{
*Correspondence: linxuepumch@qq.com; xingxp2006@126.com

${ }^{\dagger}$ Yabing Wang and Kun He contributed equally to this study as co-first authorship.

${ }^{+}$Xiaoping Xing and Xue Lin contributed equally to this study as cocorresponding authorship.

${ }^{3}$ Department of Cardiology, Peking Union Medical College Hospital, Chinese Academy of Medical Sciences \& Peking Union Medical College, Dongcheng District, Shuaifuyuan No.1, Beijing 100730, China

'Department of Endocrinology, Key Laboratory of Endocrinology of the Ministry of Health, Peking Union Medical College Hospital, Chinese Academy of Medical Science \& Peking Union Medical College, Dongcheng District, Shuaifuyuan No.1, Beijing 100730, China

Full list of author information is available at the end of the article
}

(C) The Author(s). 2020 Open Access This article is licensed under a Creative Commons Attribution 4.0 International License, which permits use, sharing, adaptation, distribution and reproduction in any medium or format, as long as you give appropriate credit to the original author(s) and the source, provide a link to the Creative Commons licence, and indicate if changes were made. The images or other third party material in this article are included in the article's Creative Commons licence, unless indicated otherwise in a credit line to the material. If material is not included in the article's Creative Commons licence and your intended use is not permitted by statutory regulation or exceeds the permitted use, you will need to obtain permission directly from the copyright holder. To view a copy of this licence, visit http://creativecommons.org/licenses/by/4.0/ The Creative Commons Public Domain Dedication waiver (http://creativecommons.org/publicdomain/zero/1.0/) applies to the data made available in this article, unless otherwise stated in a credit line to the data. 


\begin{abstract}
(Continued from previous page)
Results: Levels of serum calcium in HP and PHP were $2.05 \pm 0.16 \mathrm{mmol} / \mathrm{L}$ and $2.25 \pm 0.19 \mathrm{mmol} / \mathrm{L}$, respectively. The levels of myocardial enzyme and BNP were within the normal range. Adjusting for age at evaluation and body mass index, all M-mode measurements, left ventricular mass (LVM), LVM index (LVMI) and relative wall thickness (RWT) were comparable between patients and controls. Prolongation of corrected QT (QTC) intervals occurred in 52.6\% (10/19) of patients, and $6.7 \%(1 / 15)$ of patients manifested more than 100 episodes of supraventricular and ventricular extrasystoles, as well as supraventricular tachycardia. None of the above arrhythmias was related to a severe clinical event.
\end{abstract}

Conclusions: From this pilot study, patients diagnosed with HP and PHP and well-controlled serum calcium levels manifested normal cardiac morphology and ventricular function, except for prolonged QTc intervals, and a small percentage of mild arrhythmias needing further investigation.

Keywords: Hypoparathyroidism, Pseudohypoparathyroidism, Heart, Function, Arrhythmia

\section{Background}

Hypoparathyroidism (HP) and pseudohypoparathyroidism (PHP) are rare diseases due to parathyroid hormone (PTH) insufficiency or resistance leading to hypocalcemia, hyperphosphatemia and related symptoms. The conventional treatment for HP and PHP includes calcium supplementation combined with vitamin D analogs. To avoid hypercalciuria-related renal damage and maintain optimal levels of calcium phosphate synthesis, the serum calcium levels were regulated slightly below normal or within the low-normal range in HP patients. Hyperphosphatemia often persists among HP and PHP patients receiving conventional therapy.

High serum phosphorus directly triggers vascular injury, increases the mineral deposition in vascular smooth muscle cells in studies in vitro [1], and increases vascular calcification in individuals with or without chronic kidney disease [2], which indicates a high risk of cardiovascular diseases (CVDs) [3]. Serum calcium plays an important role in excitation-contraction coupling of the myocardium, and the influx of extracellular calcium largely initiates and determines the degree of myocardial contraction. Chronic hypocalcemia and hyperphosphatemia have been reported as risk factors for CVDs among different populations $[4,5]$. Cardiac abnormalities, such as acute heart failure, dilated cardiomyopathy, Takotsubo cardiomyopathy and arrhythmia with abnormal cardiac structure, have been reported in patients diagnosed with HP and PHP combined with chronic and severe hypocalcemia due to irregular treatment [6-8]. However, few studies have reported cardiovascular morbidities in patients with chronic HP and PHP and relatively stable levels of serum calcium [9-12]. Meanwhile, the long-term influence of chronic HP or PHP on cardiac morphology, function and conduction is still unclear, especially among patients receiving regular treatment.

Thus, the objective of the current pilot study was to directly investigate the effect of long-standing HP and PHP on cardiac morphology and function, and cardiac conductive system in patients compling with treatment of calcium and vitamin D analogs.

\section{Methods \\ Subjects and controls}

Between October 2017 and April 2018, among nearly 120 patients diagnosed with HP and PHP with a regular follow-up at the Metabolic Bone Disease Outpatient Clinic, Department of Endocrinology of Peking Union Medical College Hospital (PUMCH) in Beijing, a total of 28 subjects who agreed to participate in this study were continuously subjected to echocardiography. Patients with cardiac arrhythmia before the diagnosis of hypoparathyroidism $(n=1)$ and those diagnosed with postsurgical hypoparathyroidism due to parathyroidectomy $(n=1)$ were excluded. The remaining 26 patients and 26 age- and sex-matched healthy controls with normal thyroid function and with no CVD or associated risk factors during the same period were finally included in the analysis. The controls came from the health examination center in $\mathrm{PUMCH}$. The patients were evaluated with a 12-lead electrocardiogram (ECG), cardiac markers, echocardiography and 24-h Holter electrocardiography. The controls were evaluated with echocardiography by the same physician as the patient group. All patients received calcium combined with vitamin $\mathrm{D}$ analogs (plain vitamin D or calcitriol) at the time of investigation independently to enrollment. The study was conducted according to the principles of the Declaration of Helsinki and approved by the Ethics Committee of PUMCH. Written informed consent was obtained from all included subjects or the parents for subjects aged below 18 years. The diagnosis of HP and PHP was established by the presence of hypocalcemia, and hyperphosphatemia with inappropriately low and normal-to-high intact PTH levels, respectively, while the secondary hyperparathyroidism including chronic kidney disease and vitamin D deficiency (serum $25 \mathrm{OHD}<20 \mathrm{ng} / \mathrm{ml}$ ) [13] was excluded for PHP. All the patients had normal kidney 
function evaluated by estimated glomerular filtration rate (eGFR) and no history of chronic malabsorption or diarrhea.

\section{Clinical parameters}

Clinical demographics including diagnosis, gender, age of symptom onset, course, age of cardiac evaluation, and comorbidities (including hypertension, diabetes mellitus, and coronary artery disease (CAD)) were self-reported and confirmed by medical records. Parameters such as height, weight, and right arm systolic and diastolic blood pressure were measured by a single experienced physician. Body mass index (BMI) was calculated as weight in kilograms divided by height in squared meters. Body surface area (BSA) was calculated using the formula of $\mathrm{Du}$ Bois D and Du Bois EF [14]. Symptoms of dyspnea, shortness of breath, palpitations, history of syncope, chest pain, and edema of extremities were self-reported by patients, and evaluated and established by the same experienced physician.

\section{Laboratory parameters}

Venous blood samples were obtained in the morning after an overnight $(\geq 10 \mathrm{~h}$ ) fasting and analyzed during echocardiography and electrocardiography. The concentrations of total serum calcium $(\mathrm{Ca})$, serum phosphorus $(\mathrm{P})$, potassium, magnesium, serum $25(\mathrm{OH}) \mathrm{D}$, glucose, triglycerides, total cholesterol, low-density lipoprotein cholesterol, highdensity lipoprotein cholesterol, and creatinine were measured using an automated biochemical analyzer (Beckman Coulter AU5800, USA). Serum intact PTH was measured based on a chemiluminescence immunoassay (Siemens ADVIA Centaur, Germany). Serum P was expressed as multiples of age-specific upper limit of normal range $(\times \mathrm{ULN})$. The eGFR was calculated using the abbreviated Modification of Diet in Renal Disease Study formula [15]. Creatine kinase (CK), creatine kinase-myocardial isoenzyme (CKMB), cardiac troponin I (cTnI), and B-type natriuretic peptide (BNP) were measured simultaneously. The intra-assay coefficients of variations $(\mathrm{CVs})$ in $25(\mathrm{OH})$ $\mathrm{D}$ and PTH were 5.9 and 2.6\%, with inter-assay CVs 6.5 and $5.8 \%$, respectively. The intra-assay and inter-assay CVs of other biochemical parameters were all $<3.5 \%$. The controls self-reported that their levels of serum calcium were normal on their previous routine physical examination, which were not re-examined at the time of echocardiography examination.

\section{Echocardiography}

All the included subjects were evaluated using a noninvasive transthoracic echocardiogram (VIVID E9, GE Ultrasound, USA) with an m5s-D probe. M-mode measurements and conventional Doppler echocardiographic examinations were performed according to the
American Society of Echocardiography (ASE) guidelines [16]. The parameters of interventricular septum end-diastolic thickness (IVSd), left ventricular posterior wall end-diastolic thickness (LVPWd), left ventricular end-diastolic diameter (LVEDD), and left ventricular ejection fraction (LVEF) were measured. Early diastolic mitral inflow velocity $(E)$ and late diastolic mitral inflow velocity (A) were assessed in the Doppler-mode. Left ventricular mass (LVM, grams) was calculated using the ASE method [16]: $0.8 \times\{1.04 \text { [(IVSd + LVEDD + LVPWd })^{3}$ - $\left.\left.(\text { LVEDD })^{3}\right]\right\}+0.6$. The LVM index (LVMI, g/ $\mathrm{m}^{2}$ ) was calculated by dividing LVM by the BSA. The relative wall thickness (RWT) was calculated using the formula: $(2 \times$ LVPWd $) /$ LVEDD.

\section{Electrocardiogram}

Resting standard 12-lead ECG was obtained only in 19 patients. Resting heart rate, PR intervals, QRS duration, and corrected QT (QTc) intervals were determined via ECG analysis.

\section{4-h Holter electrocardiography}

Baseline cardiac rhythm and potential arrhythmias were evaluated using an ambulatory 12-lead electrocardiography in 15 patients over a 24-h period during their normal daily activities (MIC-Dream, JincoMed, Beijing, China). The total 24-h ventricular rates, and the maximal and minimal ventricular rates were measured automatically.

\section{Statistical analyses}

Data were analyzed using SPSS software version 22.0 (Chicago, IL, USA). Categorical variables are expressed as numbers or percentages. Continuous variables are expressed as mean \pm standard deviation or median and inter-quartile ranges, as appropriate. The clinical demographics and laboratory results of patients were compared with those of the healthy controls, and between patients diagnosed with HP and PHP using the Student's t-test or Wilcoxon test. The cardiac parameters were compared using a general linear model adjusted for age at the evaluation, gender and other confounders. Associations between serum calcium, serum phosphorus, PTH and cardiac parameters were evaluated via partial correlation analysis.

\section{Results \\ Demographic and laboratory characteristics of the 26 patients}

The study included 18 patients diagnosed with HP and eight patients with PHP. The patients with HP included three patients with secondary HP following thyroidectomy, which was defined as post-surgical HP. The average age of onset of hypocalcemic symptoms and age at the cardiac evaluation were higher in HP subjects compared with PHP subjects (age of onset $23.9 \pm 13.8$ vs. $12.9 \pm 7.4$ years, $P=$ 
Table 1 General characteristics of HP and PHP subjects

\begin{tabular}{|c|c|c|c|}
\hline Characteristic & $\mathrm{HPN}=18$ & $\mathrm{PHP} N=8$ & $P$ \\
\hline Female, n (\%) & $12(66.7 \%)$ & $7(87.5 \%)$ & 0.375 \\
\hline Age of onset (y) & $23.9 \pm 13.8$ & $12.9 \pm 7.4$ & 0.045 \\
\hline Course (y) & $22.5 \pm 15.8$ & $9.2 \pm 11.9$ & 0.046 \\
\hline Age of heart evaluation (y) & $45.4 \pm 15.4$ & $22.1 \pm 6.4$ & 0.000 \\
\hline BMI $\left(\mathrm{kg} / \mathrm{m}^{2}\right)$ & $25.1 \pm 3.9$ & $22.2 \pm 2.6$ & 0.074 \\
\hline Diabetes, $\mathrm{n}$ & 1 & 0 & 0.692 \\
\hline$C A D, n$ & 0 & 0 & NA \\
\hline Hypertension, $\mathrm{n}$ & 4 & 0 & 0.205 \\
\hline Systolic BP (mmHg) & $119.4 \pm 12.0$ & $107.6 \pm 13.8$ & 0.040 \\
\hline Diastolic BP (mmHg) & $74.4 \pm 9.0$ & $72.9 \pm 11.1$ & 0.714 \\
\hline Serum calcium (mmol/L) & $2.05 \pm 0.16$ & $2.25 \pm 0.19$ & 0.011 \\
\hline Serum phosphorus (xULN) & $1.13 \pm 0.10$ & $0.99 \pm 0.12$ & 0.003 \\
\hline Serum magnesium (mmol/L) & $0.82 \pm 0.07$ & $0.85 \pm 0.08$ & 0.464 \\
\hline Serum potassium (mmol/L) & $4.07 \pm 0.26$ & $4.10 \pm 0.31$ & 0.770 \\
\hline $25(\mathrm{OH})_{2} \mathrm{D}(\mathrm{ng} / \mathrm{mL})$ & $23.8[17.7,41.4]$ & $32.0[19.9,83.4]$ & 0.500 \\
\hline Fasting blood glucose (mmol/L) & $5.3 \pm 0.4$ & $4.9 \pm 0.4$ & 0.020 \\
\hline PTH (pg/mL) & $3[2.6,7.9]$ & $237.4[43.9482 .9]$ & 0.000 \\
\hline $\operatorname{ALP}(\times \cup L N)$ & $0.54 \pm 0.17$ & $0.71 \pm 0.37$ & 0.111 \\
\hline$\beta-C T X(\times \cup L N)$ & $0.74 \pm 0.32$ & $1.37 \pm 0.69$ & 0.008 \\
\hline eGFR (MDRD) (mL/min/1.73m²) & $90.4 \pm 21.1$ & $127.3 \pm 29.7$ & 0.005 \\
\hline Total cholesterol (mmol/L) & $4.6 \pm 0.7$ & $3.7 \pm 2.0$ & 0.178 \\
\hline Triglycerides (mmol/L) & $1.3 \pm 0.9$ & $0.7 \pm 0.6$ & 0.181 \\
\hline LDL-C (mmol/L) & $2.7 \pm 0.4$ & $2.2 \pm 1.3$ & 0.361 \\
\hline $\mathrm{HDL}-\mathrm{C}(\mathrm{mmol} / \mathrm{L})$ & $1.1 \pm 0.2$ & $1.0 \pm 0.5$ & 0.619 \\
\hline CK (U/L) & $126.5[63.8,145.8]$ & $85.0[67.5,118.3]$ & 0.153 \\
\hline CKMB $(\mu \mathrm{g} / \mathrm{L})$ & $0.40[0,0.80]$ & $0.45[0.03,0.65]$ & 0.776 \\
\hline$c T n l(\mu g / L)$ & $<0.017$ & $<0.017$ & - \\
\hline BNP (ng/L) & $13.0[8.0,25.8]$ & $6.5[3.3,11.8]$ & 0.070 \\
\hline
\end{tabular}

Date represent the means (SD), median (P25, P75), or N (\%)

Abbreviations: HP hypoparathyroidism; PHP pseudohypoparathyroidism; BMI body mass index; $C A D$ coronary artery disease; $B P$ blood pressure; $P T H$ parathyroid hormone; ALP alkaline phosphatase; $\beta$-CTX C-terminal telopeptide of type I collagen; eGFR estimated glomerular filtration rate; $L D L-C$ low-density lipoprotein cholesterol; $H D L-C$ high-density lipoprotein cholesterol; $C K$ creatine kinase; $C K M B$ creatine kinase-myocardial isoenzyme; $C T n /$ cardiac troponin I; $B N P$ B-type natriuretic peptide

0.045 ; age at cardiac evaluation $45.4 \pm 15.4$ vs. $22.1 \pm 6.4$ years, $p<0.001$, respectively). The disease courses from onset of hypocalcemia were longer in patients diagnosed with HP compared with those with PHP at the evaluation. One patient with HP suffered from diabetes, and four patients with HP had hypertension. No subject had self-reported $\mathrm{CAD}$ or related symptoms. The average levels of serum calcium were $2.05 \pm 0.16 \mathrm{mmol} / \mathrm{L}$ in $\mathrm{HP}$ patients and $2.25 \pm 0.19 \mathrm{mmol} / \mathrm{L}$ in patients with $\mathrm{PHP}(P=0.011)$ during the evaluation. Serum magnesium, serum potassium, fasting blood glucose, and lipid components were within normal limits. The serum phosphorus levels $(\times \mathrm{ULN})$ were higher $(1.13 \pm 0.10$ and $0.99 \pm 0.12, P=0.003)$ and the eGFR was lower $\left(90.4 \pm 21.1\right.$ and $127.3 \pm 29.7 \mathrm{~mL} / \mathrm{min} / 1.73 \mathrm{~m}^{2}$, $P=0.005)$ in HP than in PHP (Table 1$)$.

\section{Cardiac morphology and function in patients with HP and PHP}

The cardiac parameters of patients subjected to echocardiography were compared with age- and sex-matched healthy controls, as shown in Table 2. Adjusting for the age at evaluation and BMI, LVEF, and LVEDD, which reflect left ventricular systolic function, were comparable between patients and controls. The mean left ventricular ejection fractions were $68.4 \pm 6.4 \%$ and $66.5 \pm 7.4 \%$ in HP and PHP patients, respectively. Parameters reflecting left ventricular structure including IVSd, LVPWd, LVM, LVMI, and RWT were comparable between patients and controls. Among the 26 patients, LVMIs were less than $95 \mathrm{~g} / \mathrm{m}^{2}$ in females, and less than $115 \mathrm{~g} / \mathrm{m}^{2}$ in males. The RWT values were less than 0.42 . According 
Table 2 Comparison of echocardiographic parameters across different subgroups

\begin{tabular}{|c|c|c|c|c|c|c|c|c|c|c|}
\hline Variables & $\mathrm{HPN}=18$ & Control A N $=18$ & $\mathrm{PHP} N=8$ & Control B N=8 & $\mathrm{P}^{\mathrm{a}}$ value & $\mathrm{P}^{\mathrm{b}}$ value & $P^{c}$ value & $P^{c, d}$ & $p^{c, e}$ & $P^{c, f}$ \\
\hline Age of evaluation (y) & $45.4 \pm 15.4$ & $45.9 \pm 14.8$ & $20.6 \pm 5.3$ & $22.6 \pm 6.4$ & 0.501 & 0.120 & 0.000 & - & - & - \\
\hline BMI $\left(\mathrm{kg} / \mathrm{m}^{2}\right)$ & $25.1 \pm 3.9$ & $23.0 \pm 2.1$ & $22.6 \pm 3.3$ & $18.6 \pm 3.9$ & 0.050 & 0.004 & 0.074 & - & - & - \\
\hline \multirow[t]{2}{*}{ HR (beats/min) } & $73.6 \pm 9.9$ & $69.4 \pm 10.6$ & $70.0 \pm 4.6$ & $72.3 \pm 5.6$ & 0.269 & 0.417 & 0.404 & - & - & - \\
\hline & & & & & \multicolumn{2}{|c|}{$\begin{array}{l}\text { Adjusted for age at evaluation } \\
\text { and BMI }\end{array}$} & & & & \\
\hline IVSd (mm) & $7.6 \pm 1.1$ & $7.8 \pm 1.9$ & $6.4 \pm 0.6$ & $7.1 \pm 1.6$ & 0.600 & 0.153 & 0.009 & 0.141 & 0.159 & 0.173 \\
\hline LVPWd (mm) & $7.7 \pm 1.0$ & $7.8 \pm 1.6$ & $6.4 \pm 0.6$ & $6.5 \pm 1.4$ & 0.452 & 0.336 & 0.005 & 0.186 & 0.247 & 0.237 \\
\hline LVEDD (mm) & $46.9 \pm 5.1$ & $46.6 \pm 3.9$ & $45.1 \pm 5.0$ & $42.9 \pm 4.5$ & 0.732 & 0.836 & 0.398 & 0.124 & 0.130 & 0.14 \\
\hline LVEF (\%) & $68.4 \pm 6.4$ & $70.8 \pm 6.1$ & $66.5 \pm 7.4$ & $70.4 \pm 7.1$ & 0.275 & 0.314 & 0.422 & 0.763 & 0.939 & 0.855 \\
\hline LVM (g) & $117.9 \pm 32.1$ & $120.2 \pm 43.9$ & $86.2 \pm 20.3$ & $87.5 \pm 26.6$ & 0.517 & 0.288 & 0.020 & 0.783 & 0.888 & 0.818 \\
\hline LVMI $\left(\mathrm{g} / \mathrm{m}^{2}\right)$ & $69.2 \pm 16.0$ & $71.4 \pm 27.0$ & $54.6 \pm 6.4$ & $51.3 \pm 12.2$ & 0.644 & 0.824 & 0.050 & 0.923 & 0.943 & 0.863 \\
\hline RWT & $0.33 \pm 0.04$ & $0.34 \pm 0.07$ & $0.28 \pm 0.04$ & $0.31 \pm 0.07$ & 0.557 & 0.369 & 0.022 & 0.023 & 0.032 & 0.039 \\
\hline E/A ratio & $0.9 \pm 0.2$ & NA & $1.4 \pm 0.1$ & NA & - & - & 0.000 & 0.043 & 0.024 & 0.03 \\
\hline
\end{tabular}

Date represent the means (SD)

a: comparison between HP and control A, adjusted for BMI

b: comparison between PHP and control B, adjusted for BMI

c: comparison between HP and PHP

d: $P$ value adjusted for gender, age at evaluation, and BMI

e: $P$ value adjusted for gender, age at evaluation, $\mathrm{BMI}$ and serum calcium

$f: P$ value adjusted for gender, age at evaluation, $B M I$, and serum phosphorus

Abbreviations: HP hypoparathyroidism; PHP pseudohypoparathyroidism; BMI body mass index; HR heart rate; IVSd interventricular septum end-diastolic thickness; $L V P W d$ left ventricular posterior wall end-diastolic thickness; $L V E D D$ left ventricular end-diastolic diameter; $L V E F$ left ventricular ejection fraction; LVM left ventricular mass; LVMI left ventricular mass index; RWT relative wall thickness; $E$ early diastolic mitral inflow velocity; $A$ : late diastolic mitral inflow velocity

to the ASE guidelines, all patients displayed normal cardiac geometry (Fig. 1), except for one female patient with non-surgical HP with an RWT of 0.41 and another female with post-surgical HP with LVMI $93.2 \mathrm{~g} / \mathrm{m}^{2}$ indicating potentially borderline concentric remodeling and eccentric hypertrophy, respectively. Levels of CK, $\mathrm{CKMB}, \mathrm{cTnI}$ and BNP were within the normal ranges in both patients with HP and PHP.

\section{Comparison of echocardiographic parameters between HP and PHP patients}

Cardiac parameters reflecting left ventricular structure including IVSd, LVPWd, LVM, and RWT were significantly greater in patients with HP than in PHP, and E/A was lower in the HP group. However, after adjusting for confounders including age at evaluation, gender and BMI, only RWT and E/A showed significant differences $(P=0.023$ and $P=0.042$, respectively). Partial correlation
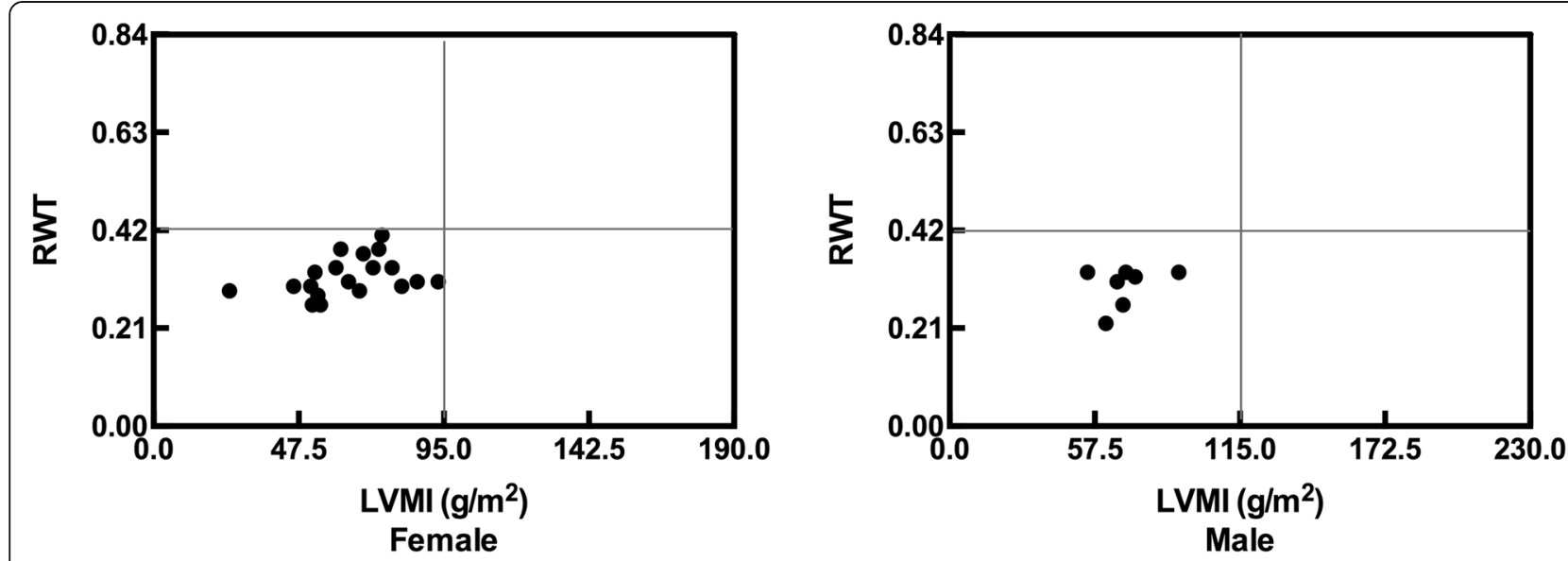

Fig. 1 Cardiac geometry of female $(n=19)$ and male $(n=7)$ patients. Normal range of RWT was $<0.42$ in both females and males, and the normal range of LVMI was $<95.0 \mathrm{~g} / \mathrm{m}^{2}$ in females and $<115.0 \mathrm{~g} / \mathrm{m}^{2}$ in males according to the American Society of Echocardiography guidelines. Abbreviations: LVMI: left ventricular mass index; RWT: relative wall thickness 
analysis showed that serum calcium, serum $\mathrm{P}$ and $\mathrm{PTH}$ were not correlated with cardiac parameters after adjusting for age at evaluation and gender (Table 2).

\section{Cardiac conduction in HP and PHP}

The resting 12-lead ECG revealed normal sinus rhythm in all patients. The average heart rate was $77.5 \pm 12.9$ beats $/ \mathrm{min}$ and $73.3 \pm 8.2$ beats $/ \mathrm{min}$ in patients with HP and PHP, respectively. Prolongation of QTc intervals was observed in 8/14 patients diagnosed with HP and in $2 / 5$ patients with PHP, with an average QTc of $473.0 \pm$ $20.3 \mathrm{~ms}$ and $455.5 \pm 6.4 \mathrm{~ms}$, respectively. In patients with $\mathrm{HP}$ and PHP, no significant differences in age of onset, disease course, levels of serum $\mathrm{Ca}$, serum $\mathrm{P}$ and serum PTH were detected among those with and without QTc prolongation. PR intervals, QRS duration and ST-T segments were all normal. Holter results revealed normal sinus rhythm in all patients, including $12 / 15$ patients (80\%) with supraventricular extrasystoles. Among the $12 / 15$ patients with supraventricular extrasystoles, one (6.7\%) female patient with non-surgical HP (aged 64 years with hypertension; disease course 26.2 years; serum $\mathrm{Ca}, 2.04 \mathrm{mmol} / \mathrm{L}$; and serum P, $1.37 \mathrm{mmol} / \mathrm{L}$ ) manifested 243 episodes of supraventricular extrasystole in $24 \mathrm{~h}$ (the incidence in the remaining 11 subjects ranged from 2 to 57 episodes), as well as supraventricular tachycardia (SVT) (52 episodes, 208 times in total) with complaints of palpitations. Among 7/15 patients (46.7\%) with ventricular extrasystoles, one $(6.7 \%)$ female patient with post-surgical HP (aged 54 years; course 41.2 years; serum $\mathrm{Ca}, 2.1 \mathrm{mmol} / \mathrm{L}$ and serum $\mathrm{P}, 1.79 \mathrm{mmol} / \mathrm{L}$ ) presented 4063 episodes of ventricular extrasystoles noticed by $24 \mathrm{~h}$ (the remaining six subjects presented with $2-16$ episodes) with shortness of breath during activity. No patient showed ventricular tachycardia. No atrioventricular block was found (Table 3).

\section{Discussion}

In this exploratory study, cardiac morphology, function and conduction were evaluated for the first time in patients with HP (mainly non-surgical HP) and PHP undergoing conventional treatment. The study demonstrated that except for mild QTc prolongation as well as a small percentage of mild arrhythmias, there was no significant difference in left ventricular morphology, systolic dysfunction, or severe cardiac events such as syncope and heart failure in patients with HP and PHP compared with ageand sex-matched healthy controls.

Previous studies suggested that both hypocalcemia and hyperphosphatemia had adverse effects on cardiovascular system $[4,5,17]$. In a recent review of 47 patients, the most common cardiac abnormalities induced by hypocalcemia were heart failure (78\%), QTc interval prolongation (38\%), and sinus tachycardia (21\%) [18]. QTc prolongation has been associated with Torsade de Pointes and sudden cardiac death $[19,20]$. In this study, although patients with HP and PHP received regular treatment and maintained low-normal to normal levels of serum calcium, a high proportion of patients $(57.1 \%$ involving HP and $40 \%$ of PHP subjects) showed QTc interval prolongation. Fortunately, arrhythmias were mild in most of our patients, and these patients had no clinical symptoms. Compared with those with severe hypocalcemia from a systemic review and meta-analysis [18], the QTc prolongation in our patients was apparently less severe $(449.9 \pm 33.9 \mathrm{~ms}$ in HP and $440.6 \pm 16.2$ $\mathrm{ms}$ in PHP vs. $510 \mathrm{~ms}$ (median) reported in the literature), and the average level of serum calcium in our

Table 3 Cardiac conduction in patients with HP and PHP

\begin{tabular}{lll}
\hline Variables & $H P$ & PHP \\
\hline 12-lead ECG & $N=14$ & $N=5$ \\
HR (beats/min) & $77.5 \pm 12.9$ & $73.3 \pm 8.2$ \\
QTc interval (ms) & $449.9 \pm 33.9$ & $440.6 \pm 16.2$ \\
QTc abnormality, n (\%) & $8 / 14(57.1 \%)$ & $2 / 5(40 \%)$ \\
QT (ms) & $401.3 \pm 33.4$ & $392.5 \pm 14.3$ \\
QRS interval (ms) & $87.5 \pm 11.1$ & $92.5 \pm 15.4$ \\
PR interval (ms) & $145.9 \pm 18.1$ & $146.5 \pm 10.5$ \\
24-h Holter ECG & $N=10$ & $N=5$ \\
24-h total HR (beats) & $110,428.0 \pm 12,472.5$ & $108,930 \pm 11,826.4$ \\
Highest HR (beats/min) & $122.8 \pm 18.3$ & $136.4 \pm 12.9$ \\
Lowest HR (beats/min) & $55.9 \pm 3.9$ & $57.2 \pm 10.7$ \\
Number of supraventricular arrhythmias, $\mathrm{n}$ & $10 / 10$ & $2 / 5$ \\
Number of ventricular arrhythmia, $\mathrm{n}$ & $5 / 10$ & $2 / 5$
\end{tabular}

Date represent the means (SD) or $\mathrm{N}(\%)$

Abbreviation: HP hypoparathyroidism; PHP pseudohypoparathyroidism; ECG electrocardiograms; $H R$ heart rate 
patients was higher $(2.05 \pm 0.16 \mathrm{mmol} / \mathrm{L}$ in $\mathrm{HP}$ and $2.25 \pm 0.19 \mathrm{mmol} / \mathrm{L}$ in $\mathrm{PHP}$ vs. $1.36 \mathrm{mmol} / \mathrm{L}$ (median) in published values).

In addition, supraventricular and ventricular extrasystoles were detected in $80 \%$ and $46.7 \%$ of our subjects evaluated by 24-h Holter electrocardiography. Two patients presented with palpitations or shortness of breath accompanied by supraventricular tachycardia or frequent ventricular extrasystoles. Studies found that ventricular extrasystoles, supraventricular extrasystoles, and supraventricular tachycardia occurred in $40-75 \%, 60.8 \%$, and $2.2 \%$ respectively in healthy subjects via $24-$ to $48 \mathrm{~h}$ Holter examination [21-24]. Even frequent ventricular extrasystoles (more than 60 beats per hour) have been reported in apparently healthy persons [25]. However, evidence suggested that even frequent ventricular extrasystoles did not increase mortality. Kennedy et al. [23] found the risk of death in asymptomatic healthy subjects ( $n=73$, mean age $46.0 \pm 13.3$ years; $32 \%$ with hypertension) with frequent and complex ventricular extrasystoles $(63 \%)$ for $6.5 \pm 1.8$ years (from 1 to 9.5 years) was not increased according to US death rates during the same period. In another study, Maurer et al. [26] found that exercise-induced supraventricular tachycardia $(6.0 \%$ in males and $6.3 \%$ in females) in apparently healthy volunteers (843 males and 540 females, aged 20-94 years) did not increase the risk of cardiovascular mortality or coronary events during a follow-up of $5.7 \pm 3.9$ years compared with control subjects matched for age and sex. However, subjects with SVT appeared to show a higher incidence of spontaneous supraventricular tachyarrhythmia and a higher rate of lone atrial fibrillation than the controls. Due to the small sample size and cross-sectional analysis of this study, it was difficult to assess the severity of arrhythmia in patients with HP and PHP. Thus, further observations and close monitoring are needed to investigate the severity of arrhythmia and related factors in a larger sample size with long-term follow-up.

In this case-control study, no significant differences in left ventricular structure and systolic function were observed between HP and PHP patients with low-normal or normal levels of serum calcium and healthy controls, based on echocardiography. Left ventricular hypertrophy was not observed in all patients, although a few female patients may display borderline concentric remodeling and eccentric hypertrophy, as shown in Fig. 1. An early and small study reported cardiac function in eight HP/PHP patients (aged 13-31 years) with symptomatic hypocalcemia (average serum calcium $1.8 \mathrm{mmol} / \mathrm{L}$; range, $1.33-2.13$ $\mathrm{mmol} / \mathrm{L}$ ), and normal M-mode measurements with unknown LVMI and RWT [9]. Acute changes in circulating ionized calcium levels altered myocardial contractility in experimental studies [27]. Clinical studies reported various cardiac events, including acute heart failure and abnormal cardiac structure and function, in HP and PHP patients with severe hypocalcemia (serum calcium range, 0.78$1.55 \mathrm{mmol} / \mathrm{L}$ ) [28-30]. Hypocalcemia (range, 0.65-1.90 $\mathrm{mmol} / \mathrm{L})$ was significantly correlated with $\operatorname{LVEF}(\mathrm{B}=5.16$, $P<0.01$ ) in patients with HP [18]. LVEF was normal in patients with hypocalcemia and was not correlated with serum calcium levels probably due to the limited sample size and fewer comorbidities associated with cardiovascular risk. We speculated that the acute and severe hypocalcemia may have different impact on cardiac function due to chronic sub-normal hypocalcemia in relatively wellcontrolled hypoparathyroidism in this study.

Compared with PHP patients, RWT appeared to be larger in HP patients in our study after adjusting for age at evaluation, BMI, and levels of serum calcium and serum phosphorus. However, they were all within the normal range. The levels of serum calcium, serum phosphorus, and PTH were not related to RWT. In addition, due to the small sample size, patients with post-surgical and nonsurgical HP were not separated in our study. Therefore, further classification of large samples of patients diagnosed with HP and PHP into subgroups for comparative analyses may facilitate the determination of differences and clinical significance accurately.

This study has limitations. The major limitation is the small sample size due to the rarity of non-surgical HP and PHP. The cross-sectional design is another limitation, which prevented the analysis of changes in cardiac structure and function during long-term follow-up and precluded any inferences of causal relationship. Also, we have not included patients with previous heart damage due to hypocalcemia, and therefore, the cardiac characteristics of such patients may have been missed. We only included patients who consented to cardiac examination, which suggested a selection bias. Another possible weakness is the relative youth of the included patients since the effects of HP on the cardiovascular system take longer and may be more dramatic in older patients. Meanwhile, date from the controls including biochemical indicators, 12 lead ECG and 24-h Holter electrocardiography were incomplete, although we speculated that the results of the study were not significantly affected. Finally, the patients were not categorized into subgroups according to different etiologies and the small sample size prevented any comparison of differences between subgroups.

\section{Conclusions}

The results of this preliminary study indicate no definitive evidence of a negative effect of cardiac structure or systolic function among patients diagnosed with HP and PHP receiving regular calcium and vitamin D analogs, except for a significant proportion of subjects with 
prolonged QTc and a few subjects of arrhythmia, warranting further investigation. Studies with a larger sample size are warranted to verify or further explore this situation.

\begin{abstract}
Abbreviations
A: Late diastolic mitral inflow velocity; ASE: American Society of Echocardiography; BMI: Body mass index; BNP: B-type natriuretic peptide; BSA: Body surface area; Ca: Calcium; CAD: Coronary artery disease; CK: Creatine kinase; CKMB: Creatine kinase-myocardial isoenzyme; cTnl: Cardiac troponin I; CVDs: Cardiovascular diseases; E: Early diastolic mitral inflow velocity; ECG: Electrocardiogram; eGFR: Estimated glomerular filtration rate; HP: Hypoparathyroidism; IVSd: Interventricular septum end-diastolic thickness; LVEDD: Left ventricular end-diastolic diameter; LVEF: Left ventricular ejection fraction; LVPWd: Left ventricular posterior wall enddiastolic thickness; P: Phosphorus; PHP: Pseudohypoparathyroidism; PTH: Parathyroid hormone; PUMCH: Peking Union Medical College Hospital; QTc: Corrected QT; RWT: Relative wall thickness; SVT: Supraventricular tachycardia; ULN: Upper limit of normal range
\end{abstract}

\section{Acknowledgments}

We thank the patients and staff of who participated in this study. We thank Dr. Yongjun Wang and Dr. Yan Jia for booking 12-lead electrocardiogram, echocardiography and 24-h Holter electrocardiography examination for the subjects in this study. We thank LetPub (www.letpub.com) for its linguistic assistance during the preparation of this manuscript. The abstract of this manuscript has been previously presented at the conference of European Calcified Tissue Society in 2019 as a poster, and the 11th Youth conference of Chinese Society of Bone and Mineral Research as a poster.

\section{Authors' contributions}

YBW, KH: data acquisition, analysis and interpretation of the data, and drafting of the manuscript; OW, XL, SXC, YJ, ML, WBX, XPX: data acquisition. $\mathrm{OW}, \mathrm{XL}, \mathrm{XPX}$ : study concept and design, critical revision of the manuscript. All authors read and approved the final manuscript.

\section{Funding}

This study was supported by the National Natural Science Foundation of China (No. 81873641 and No.81670349). These two funds financed the physician's labor fee for 12-lead electrocardiogram, echocardiography and 24-h Holter electrocardiography examination of subjects in this study, as well as the embellishment fee of this manuscript.

\section{Availability of data and materials}

The datasets generated during and/or analysed during the current study are available from the corresponding author on reasonable request.

\section{Ethics approval and consent to participate}

The study was approved by the Ethics Committee of Peking Union Medical College Hospital. Written informed consent was obtained from all included subjects or the parents for subjects aged below 18 years.

\section{Consent for publication}

Not applicable.

\section{Competing interests}

The authors declare that they have no competing interests.

\section{Author details}

${ }^{1}$ Department of Endocrinology, Key Laboratory of Endocrinology of the Ministry of Health, Peking Union Medical College Hospital, Chinese Academy of Medical Science \& Peking Union Medical College, Dongcheng District, Shuaifuyuan No.1, Beijing 100730, China. ${ }^{2}$ Department of Internal Medicine, Peking Union Medical College Hospital, Chinese Academy of Medical Sciences \& Peking Union Medical College, Dongcheng District, Shuaifuyuan No.1, Beijing 100730, China. ${ }^{3}$ Department of Cardiology, Peking Union Medical College Hospital, Chinese Academy of Medical Sciences \& Peking Union Medical College, Dongcheng District, Shuaifuyuan No.1, Beijing 100730, China.
Received: 30 May 2019 Accepted: 28 April 2020

Published online: 11 May 2020

\section{References}

1. Jono S, McKee MD, Murry CE, Shioi A, Nishizawa Y, Mori K, Morii H, Giachelli CM. Phosphate regulation of vascular smooth muscle cell calcification. Circ Res. 2000;87:E10-7.

2. Giachelli CM. Ectopic calcification: gathering hard facts about soft tissue mineralization. Am J Pathol. 1999;154:671-5.

3. Latif F, Khalid MM, Khan F, Omar Z, Ali FA. Role of hyperphosphatemiamediated vascular calcification in cardiovascular outcomes and its management: a review. J Cardiovasc Med (Hagerstown). 2013;14:410-5.

4. Yarmohammadi H, Uy-Evanado A, Reinier K, Rusinaru C, Chugh H, Jui J, Chugh SS. Serum calcium and risk of sudden cardiac arrest in the general population. Mayo Clin Proc. 2017;92:1479-85.

5. Palmer SC, Hayen A, Macaskill P, Pellegrini F, Craig JC, Elder GJ, Strippoli GF. Serum levels of phosphorus, parathyroid hormone, and calcium and risks of death and cardiovascular disease in individuals with chronic kidney disease: a systematic review and meta-analysis. JAMA. 2011;305:1119-27.

6. Cakerri L, Husi G, Minxuri D, Roko E, Vyshka G. Primary hypoparathyroidism presenting with heart failure and ventricular fibrillation. Oxf Med Case Reports. 2014:2014:77-9.

7. Rhee HS, Lee SW, Jung YK, Jeon U, Park SH, Lee SJ, 321 Sin WY, Jin DK. Takotsubo cardiomyopathy associated with severe hypocalcemia secondary to idiopathic hypoparathyroidism. Korean Circ J 2013;43: 573-577.

8. Rentoukas E, Lazaros G, Sotiriou S, Athanassiou M, Tsiachris D, Deftereos S, Stefanadis C. Extreme but not life-threatening QT interval prolongation? Take a closer look at the neck! J Electrocardiol. 2013:46:128-30.

9. Vered I, Vered Z, Perez JE, Jaffe AS, Whyte MP. Normal left ventricular performance documented by Doppler echocardiography in patients with long-standing hypocalcemia. Am J Med. 1989;86:413-6.

10. Vadiveloo T, Donnan PT, Leese CJ, Abraham KJ, Leese GP. Increased mortality and morbidity in patients with chronic hypoparathyroidism: a population-based study. Clin Endocrinol. 2019;90:285-92.

11. Underbjerg L, Sikjaer T, Mosekilde L, Rejnmark L. Cardiovascular and renal complications to postsurgical hypoparathyroidism: a Danish nationwide controlled historic follow-up study. J Bone Miner Res. 2013; 28:2277-85

12. Underbjerg $L$, Sikjaer T, Mosekilde $L$, Rejnmark $L$. Pseudohypoparathyroidism - epidemiology, mortality and risk of complications. Clin Endocrinol. 2016;84: 904-11.

13. Rosen CJ, Abrams SA, Aloia JF, Brannon PM, Clinton SK, Durazo-Arvizu RA, Gallagher JC, Gallo RL, Jones G, Kovacs CS, Manson JE, Mayne ST, Ross AC, Shapses SA, Taylor CL. IOM committee members respond to Endocrine Society vitamin D guideline. J Clin Endocrinol Metab. 2012;97:1146-52.

14. Du Bois D, Du Bois EF. A formula to estimate the approximate surface area if height and weight be known. 1916. Nutrition. 1989;5:303-11 discussion 312-313.

15. Levey AS, Coresh J, Greene T, Stevens LA, Zhang YL, Hendriksen S, Kusek JW, Van Lente F. Chronic Kidney Disease Epidemiology Collaboration. Using standardized serum creatinine values in the modification of diet in renal disease study equation for estimating glomerular filtration rate. Ann Intern Med. 2006;145:247-54

16. Lang RM, Badano LP, Mor-Avi V, Afilalo J, Armstrong A, Ernande L, Flachskampf FA, Foster E, Goldstein SA, Kuznetsova T, et al. Recommendations for cardiac chamber quantification by echocardiography in adults: an update from the American Society of Echocardiography and the European Association of Cardiovascular Imaging. J Am Soc Echocardiogr. 2015;28:1-39 e14.

17. Wang Y, Ma H, Hao X, Yang J, Chen Q, Lu L, Zhang R. Low serum calcium is associated with left ventricular systolic dysfunction in a Chinese population with coronary artery disease. Sci Rep. 2016;6:22283.

18. Newman DB, Fidahussein SS, Kashiwagi DT, Kennel KA, Kashani KB, Wang Z, Altayar O, Murad MH. Reversible cardiac dysfunction associated with hypocalcemia: a systematic review and meta-analysis of individual patient data. Heart Fail Rev. 2014;19:199-205.

19. Moss AJ. The QT interval and torsade de pointes. Drug Saf 1992;21(Suppl 1): 5-10; discussion 81-7.

20. Straus SM, Kors JA, De Bruin ML, van der Hooft CS, Hofman A, Heeringa J, Deckers JW, Kingma JH, Sturkenboom MC, Stricker BH, et al. Prolonged QTC 
interval and risk of sudden cardiac death in a population of older adults. $J$ Am Coll Cardiol. 2006;47:362-7.

21. Ng GA. Treating patients with ventricular ectopic beats. Heart. 2006;92 1707-12.

22. Hiss RG, Lamb LE. Electrocardiographic findings in 122,043 individuals. Circulation. 1962;25:947-61.

23. Kennedy HL, Whitlock JA, Sprague MK, Kennedy LJ, Buckingham TA, Goldberg RJ. Long-term follow-up of asymptomatic healthy subjects with frequent and complex ventricular ectopy. N Engl J Med. 1985;312:193-7.

24. Hingorani P, Karnad DR, Rohekar P, Kerkar V, Lokhandwala YY, Kothari S. Arrhythmias seen in baseline 24-hour Holter ECG recordings in healthy Normal volunteers during phase 1 clinical trials. J Clin Pharmacol. 2016;56: 885-93

25. Kennedy HL, Underhill SJ. Frequent or complex ventricular ectopy in apparently healthy subjects: a clinical study of 25 cases. Am J Cardiol. 1976; 38:141-8.

26. Maurer MS, Shefrin EA, Fleg JL. Prevalence and prognostic significance of exercise-induced supraventricular tachycardia in apparently healthy volunteers. Am J Cardiol. 1995;75:788-92.

27. Drop L, Geffin GA, O'Keefe DD, Newell JB, Jacobs ML, Fowler BN, Daggett WM. Relation between ionized calcium concentration and ventricular pump performance in the dog under hemodynamically controlled conditions. Am J Cardiol. 1981;47:1041-51.

28. Vlot $\mathrm{M}$, de Jong $\mathrm{M}$, de Ronde $\mathrm{P}$, Tukkie R. A surprising cause of reversible dilated cardiomyopathy. BMJ Case Rep. 2014;2014. https://doi.org/10.1136/ bcr-2013-203512.

29. Suzuki T, Ikeda U, Fujikawa H, Saito K, Shimada K. Hypocalcemic heart failure: a reversible form of heart muscle disease. Clin Cardiol. 1998; 21:227-8

30. Sussman RM, Edinburgh A. Chronic idiopathic hypoparathyroidism simulating cardiac asthma. N Engl J Med. 1957;256:734-9.

\section{Publisher's Note}

Springer Nature remains neutral with regard to jurisdictional claims in published maps and institutional affiliations.

Ready to submit your research? Choose BMC and benefit from:

- fast, convenient online submission

- thorough peer review by experienced researchers in your field

- rapid publication on acceptance

- support for research data, including large and complex data types

- gold Open Access which fosters wider collaboration and increased citations

- maximum visibility for your research: over $100 \mathrm{M}$ website views per year

At $\mathrm{BMC}$, research is always in progress.

Learn more biomedcentral.com/submissions 